\title{
211
}

Coggins $\mathrm{J}^{1}$, Cartwright $\mathrm{R}^{2}$, Bergmann $\mathrm{J}^{3}$

1. Chiaro Technology Ltd., 2. Faculty of Medicine, School of Public Health, Imperial College London, 3. The Institute of Biomedical Engineering, University of Oxford

\section{MEASURES OF PELVIC FLOOR STRENGTH BY AGE AND PARITY USING THE ELVIE DEVICE}

\section{Hypothesis / aims of study}

Elvie is a newly commercially available intra-vaginal pelvic floor exercise device (see Figure 1). It contains a force-sensitive resistor (FSR) and accelerometer, to simultaneously measure pelvic floor contraction and displacement. Realtime biofeedback is available to the user, and time series measures of pelvic floor strength are stored. The device has potential as an adjunct or alternative to supervised pelvic floor muscle training (PFMT), and might also be a useful method of outcome data collection in clinical trials of PFMT interventions. Using data from a large cohort of users of the device, we aimed to measure pelvic floor strength before training, and test the impact of age and childbirth on pelvic floor contraction, with the overall goal of assessing the Elvie device as a research tool.

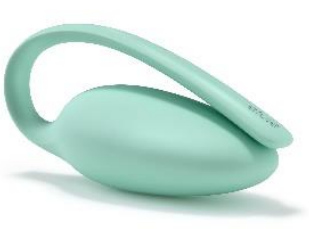

Figure 1: Elvie device

\begin{tabular}{|l|l|l|l|l|}
\hline Age & Nulliparous & Primiparous & Multiparous & Total \\
\hline $\mathbf{3 0 - 3 9}$ & 211 & 143 & 169 & 523 \\
\hline $\mathbf{4 0 - 4 9}$ & 93 & 70 & 255 & 418 \\
\hline $\mathbf{5 0 - 5 9}$ & 49 & 28 & 164 & 241 \\
\hline Total & 353 & 241 & 588 & 1182 \\
\hline
\end{tabular}

Table 1. Counts for segmented users

\section{Study design, materials and methods}

We used a fully anonymised data extract from the Elvie database, including all Elvie users who have used the device, and paired it with the app. Each device is individually calibrated during manufacture, to provide a measure of pelvic floor contraction in newtons. To use Elvie, the user inserts the device into the vagina, with the tail remaining outside. A Bluetooth connection is established between Elvie and the user's phone or tablet. The Elvie app then guides the user through a series of exercises. Force and accelerometer data are serially recorded at a frequency of $10 \mathrm{~Hz}$. The pitch of the device is assessed from accelerometer data, to determine if the user is supine or upright. We analysed data from an exercise in which the user is prompted to relax her pelvic floor muscle for three seconds, and then contract to the peak of her ability for three seconds. Age and parity were selfreported using on-screen questions in the app. We categorised age into 10 year intervals (30-39, 40-49, 50-59), and categorised parity as nulli-, primi-, and multiparous. A Box-Cox power transform applied to force data resulted in distributions not significantly different from normal ( $p>0.10$, tested via the Anderson-Darling test), and thus we assessed statistical significance of the difference in samples via Student's $t$-test applied to transformed samples. We used Bonferroni correction for multiple testing. To test for differences according to parity, we computed the difference in medians between parity segments whilst keeping the age category constant, and reversed this procedure to test differences among age groups. We further tested for age relationships by regressing transformed data against age as a continuous variable, using a linear least-squares method. We assessed significance via a Student's $t$-test, employing White's heteroskedasticity-consistent standard error.

\section{Results}

After data cleaning, we had a sample size of 1182 users, of whom we could determine orientation for 853 users (260 upright, 593 supine) (see Table 1). Distributions of the relaxed state and peak contraction are displayed in Figure 2(a) and (b), respectively. The median value for the relaxed state is $0.27 \mathrm{~N}$ whilst supine and $0.51 \mathrm{~N}$ whilst upright $(p<0.001)$. For the peak contraction, the median value whilst supine is $0.40 \mathrm{~N}$ and whilst upright is $0.69 \mathrm{~N}(p<0.001)$. Median values for the segmentation by age and parity are shown in Figure 3. For the relaxed state, we find a significant decrease in force $(-0.11 \mathrm{~N}, p=0.01)$ for the age bracket $30-39$ between multiparous and nulliparous women. For the peak contraction, in the 30-39 age bracket, we also find a significant decrease in force between primiparous and nulliparous $(-0.48, p<0.001)$, and multiparous and nulliparous women $(-0.39$, $p<0.001)$. In the 40-49 bracket, significant differences in force occur between multiparous and nulliparous women $(-0.44, p=0.005)$ and between multiparous and primiparous women $(-0.26, p<0.001)$. No significant changes in samples are observed between parity segments for the 50-59 age bracket. The only significant relationship found by testing among age groups (whilst maintaining constant parity) was an increase in the median peak contraction for primiparous women in the age brackets of 40-49 and 30-39 $(0.34, p<0.001)$. For peak contraction, we found a significant positive regression slope for primiparous women $(p=0.03)$, and a significant negative slope for multiparous women $(p=0.04)$.

\section{Interpretation of results}

Significant relationships are observed between exercise orientations for both the relaxed state and peak contraction, in contrast to earlier evaluations using balloon catheters [1]. This indicates that the base intra-vaginal force is higher when upright, and may also indicate that the user is better able to isolate the pelvic floor whilst standing. Relationships between intra-vaginal force and segments are more apparent for the peak contraction than for the relaxed state, and appear to be stronger for parity than age. However, the parity relationships appear to become less important as the user ages, consistent with previous evidence of attenuation of impacts of childbirth on stress incontinence with age [2]. The significant increase in peak contraction for primiparous women might suggest a recovery period post-partum, with younger women being more likely to have given birth recently. Previous evidence regarding any effect of age is inconsistent [3]. Any effect on the relaxed state may be below the current noise level of the device or missed as a consequence of the way this sample was assembled. 
(a)

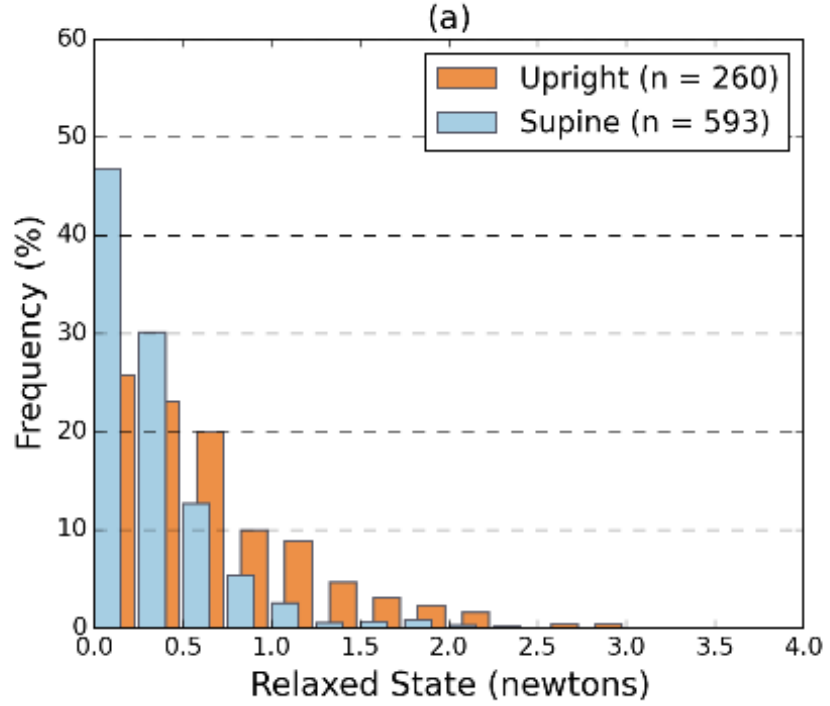

(b)

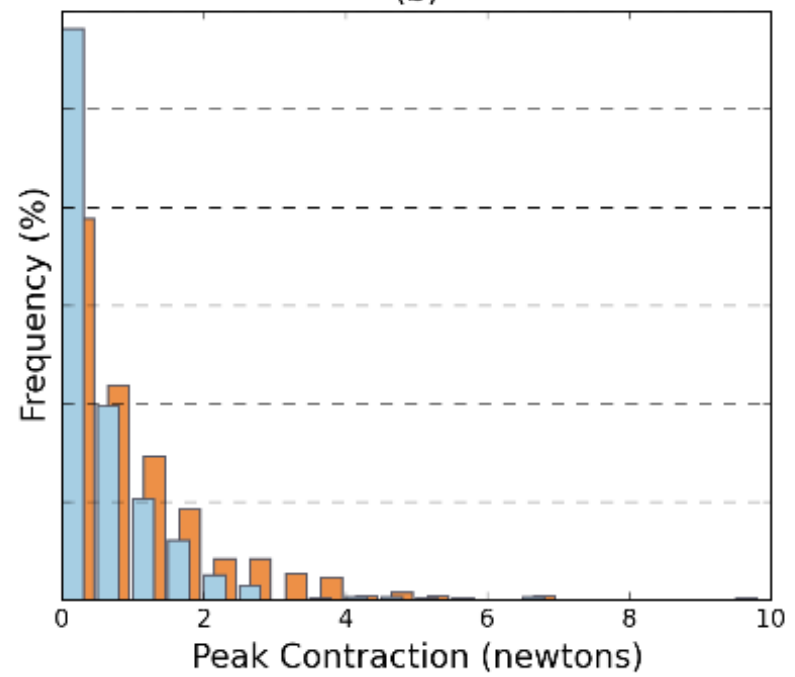

Figure 2: Distributions of relaxed state force (a) and peak contraction force (b)
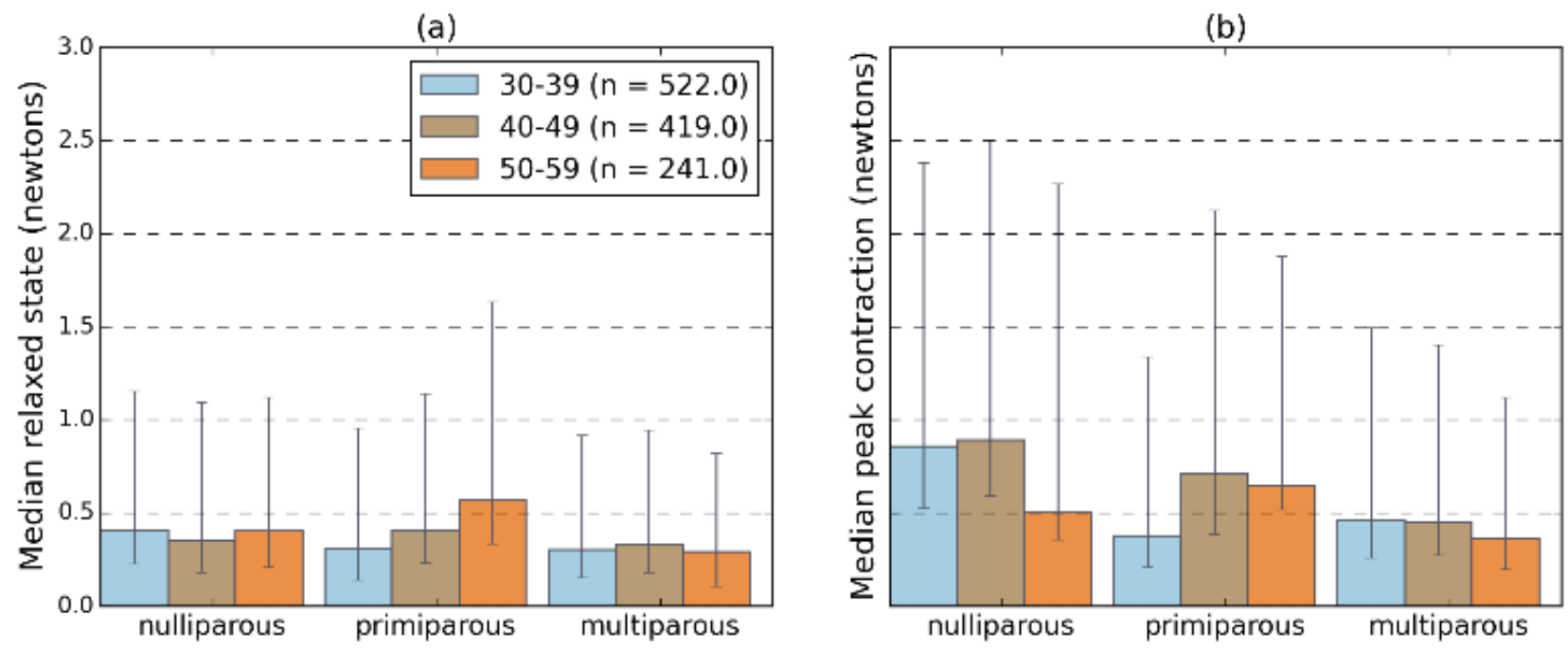

Figure 3: Median values of relaxed state (a) and peak contraction (b), by age and parity. Error bars show inter-quartile range.

\section{Concluding message}

Using a large sample of users of a commercially available pelvic floor fitness device, we rigorously assessed pelvic floor strength and its correlates. We identified and quantified highly significant impacts on pelvic floor strength from childbirth, which were attenuated with age. Elvie has potential to provide a wealth of information on pelvic floor strength, both for measuring clinical training benefits and in future clinical trials.

References

1. Acta Obstet Gynecol Scand. 2003 Dec;82(12):1120-4

2. Eur Urol. 2016 Feb 10. pii: S0302-2838(16)00156-1. doi: 10.1016/j.eururo.2016.01.037

3. BMC Urol. 2015 Apr 10;15:29. doi: 10.1186/s12894-015-0017-6.

Disclosures

Funding: Jack Coggins is a scientist employed by Chiaro Technology Ltd., the maker of Elvie. Clinical Trial: No Subjects: HUMAN Ethics not Req'd: data were routinely collected and fully anonymised. Helsinki: Yes Informed Consent: Yes 